\title{
Numerical modelling of a dual-rotor marine current turbine in a rectilinear tidal flow
}

\author{
Kaiming $\mathrm{Ai}^{\mathrm{a}}$, Jiahuan Cui ${ }^{\mathrm{b}}$, Mingyang Wang ${ }^{\mathrm{a}}$, Eldad Avital ${ }^{\mathrm{a}, *}$ \\ ${ }^{a}$ School of Engineering and Materials Science, Queen Mary University of London, UK \\ ${ }^{b}$ ZJU-UIUC, International campus, Zhejiang University, China
}

\begin{abstract}
In this study, numerical simulation is used to investigate a counter-rotating dual-rotor marine current turbine (MCT) that is aligned for a rectilinear tidal current. Results of power and thrust coefficients and the mean wake axial velocity are compared with that of the blade element momentum (BEM) method coupled with the Park wake model. For a single-rotor MCT, small discrepancies are observed for front rotor, and larger discrepancies for rear rotor when comparing the $\mathrm{CFD}$ and BEM results. The mean axial wake velocity agrees better with the higher turbulence intensity (TI). CFD results shows that the power coefficient $\left(C_{P}\right)$ of rear rotor depends on the ambient turbulence intensity. The maximum $C_{P}$ of dual-rotor turbine is $5 \%$ higher than that of just the front rotor. Streamlines show that a large vortex is formed behind the rear rotor. The numerical simulations give more credibility to the BEM Park model, but also points to its sensitivity to the incoming turbulence intensity.
\end{abstract}

Keywords: Marine current turbine, Dual-rotor, Counter-rotating, CFD, Bi-directional

2019 MSC: 00-01, 99-00

\section{Introduction}

The blades of horizontal axis marine current turbine (MCT) are normally installed at a pitch angle so that the turbine can be operating at the optimal angle of attack $(A O A)$. For wind application this may require yaw control to adjust for the varying wind direction, but for marine currents with known and steady stream direction, a yaw-free control horizontal axis MCT seems to be ideally suited [1]. However, the tidal current can alter its velocity direction opposite during the day, i.e. a rectilinear current. This will leave the horizontal axis MCT blades pitched at wrong angle for the flow coming from the opposite

\footnotetext{
* Corresponding author

Email addresses: k.ai@qmul.ac.uk (Kaiming Ai), jiahuancui@intl.zju.edu.cn (Jiahuan Cui), mingyang.wang@qmul.ac.uk (Mingyang Wang), e.avital@qmul.ac.uk (Eldad Avital)
}

Preprint submitted to Elsevier

December 3, 2019 
direction, causing possible stall and much reduced power. This can be mitigated using the pitch angle control as commonly used in the wind turbine. However, the pitch controlled turbine increases the manufacturing cost and reduces the reliability. Due to higher waterproof standard, the electrical pitch system for tidal turbine is more expensive and vulnerable than that of a wind turbine which

15 normally operates in a dry environment [2]. Thus, the industrial application of the pitch control system is commonly used for very large turbines and not for small and medium size ones. One possible solution for the bi-directional current flow is the dual-rotor configuration with fixed pitch as illustrated in Fig. 11a. Such dual-rotor configuration has two rotors facing towards opposite directions.

${ }_{20}$ Regardless of the current direction, the power output of a dual-rotor should be the same. Relative to the flow direction, the upstream blade is named as the front rotor and the downstream blade is the rear rotor. To maintain higher efficiency, the front and rear rotors are normally rotating in opposite directions.

In 1983, Newman pursued an analytical study on a dual-disc kinetic turbine based on the actuator disc theory, and concluded that the maximum power coefficient could be 16/25 when both rotors are aligned for a unidirectional flow, which is $8 \%$ higher than Betz limit (16/27) [3. Three years later, Newman extended his dual-disc model to multiple actuator-disc model, and concluded that the optimal $C_{P}$ is $8 n(n+1) /\left[3(2 n+1)^{2}\right]$ for $n$ disc in tandem. The maximum

30 $C_{P}$ is $2 / 3$, which is $13 \%$ higher than the maximum $C_{P}$ of the single actuator disk model [4. A more recent update of the dual-disc model was done by Sundararaju [5], who proposed no assumption on the flow pressure between the rotors. Based on his model, the maximum $C_{P}$ is 0.814 with a rotor spacing of 2.8 times of rotor diameter. Later, the performance of a counter rotating wind turbine (CRWT) 35 was evaluated for unidirectional wind using more advanced methods, including the blade element momentum (BEM) theory, actuator line model [6, 7], freewake vortex lattice method [8] and CFD 7 . The wake velocity behind the front rotor is evaluated using the experimental wake data [9] or wake models. Several in-site experiments on CRT prototypes [9, 10, 11] were conducted for

40 the validation of theoretical and numerical results. Parametric studies including pitch angles [12, rotor spacings [5], rotor diameter ratios [9], and rotational speed control for rotors have been reported. Cho [10] proposed an integrated control algorithm for a counter-rotating dual rotor/dual generator wind turbine configuration. For a CRT operating in an isolated environment, the full-scale 45 prototype tests 9, 10] showed that the maximum $C_{P}$ of a CRWT was close to 0.5. For a wind farm, the numerical results from Vaselbehagh [7] showed that the dual-rotor turbines produced $22.6 \%$ more power than the single-rotor turbines.

In the field of a counter rotating MCT that operates in a unidirectional 50 current, Charke [13] and Huang [14] pursued numerical and experimental tests for rotors with a small rotor spacing $(X / D<0.5, X$ is the rotor spacing, and $D$ is the rotor diameter). Charke used a modified BEM theory to evaluate the performance of the turbine, and conducted a towing tank test, resulting a peak $C_{P}$ of 0.39 . Huang designed a counter rotating turbine composed of ${ }_{55}$ NACA0015 and MEL002 hydrofoil with rotor spacing of 0.1D. However, a poor 
agreement on $C_{P}$ was observed between the numerical and experimental results. Meanwhile, a scattered $C_{P}$ was observed in the experimental results.

The above research provides valuable guideline for our design of a counter rotating dual-rotor MCT, where the front rotor is pitched towards upstream and the rear rotor is pitched towards downstream for a rectilinear tidal current. This configuration is different from that of counter-rotating turbines mentioned above, where both rotors are pitched towards the same direction. Huang [15] designed a dual-rotor counter rotating horizontal axis MCT pitched in opposite direction with a close rotor spacing $(X / D<0.5)$. However, the overall $C_{P}$ of 65 the dual-rotor turbine was still below 0.4 .

In our previous study [16, the dual-rotor MCT was analyzed using the BEM-Park model. Numerical results showed that the $C_{P}$ of dual-rotor MCT could be as high as 0.41 at $R e_{c}=135 k$ with the NACA0012 blade profile, and 0.55 if $R e_{c}=1 M$ with the NACA0018 blade profile. However, the Park wake model is a simplified model with several assumptions. More advanced models are needed to better predict the performance of the rear rotor blade. The aim of the current study is to study the dual-rotor performance using the CFD method. The rotor configuration and numerical methodology are outlined in the next section, followed by comparison between the BEM-Park model and

75 CFD results. A brief summary is presented at the end.

\section{Turbine geometry}

The three-blade horizontal axis MCT of Luznik 17. was used as the base geometric configuration. The original E387 blade profile was replaced by the symmetric NACA0012 profile. This is because as was found in [16] using the

so BEM-Park model there was no benefit operating a rear rotor composed of the E387 profile due to the highly negative power in the rear rotor. This means the rear rotor consumes power instead of producing power because of its very high negative angle of attack caused by the negative camber. To achieve optimal performance in terms of $C_{P}$ for high $T S R$, the blades were twisted at angles 85 varying with respect to the local radius, i.e. $\theta=\theta_{T} /(r / R)$ [18. Here, $\theta_{T}$ represents the blade pitch angle at the tip, $r$ is the radial distance from the hub, and $R$ is the rotor's radius. This is because the tangential velocity at the tip is much higher than in the hub. To keep the local angle of attack below stall, the pitch angle at the tip is much lower than in the hub. Fig. 22 (a) illustrates the 90 pitch angle of the NACA0012 blades investigated in the current work, with the tip pitched at 2, 0 and -2 degrees respectively. Different from the pitch angle, the chord length distribution follows a linear variation along the spanwise direction as presented in Fig. 2 (b).

For performance and load analysis of a horizontal axis MCT, the three basic terms are tip speed ratio, power coefficient, and thrust coefficient, which are defined as;

$$
T S R=\frac{\Omega R}{U_{\infty}}
$$




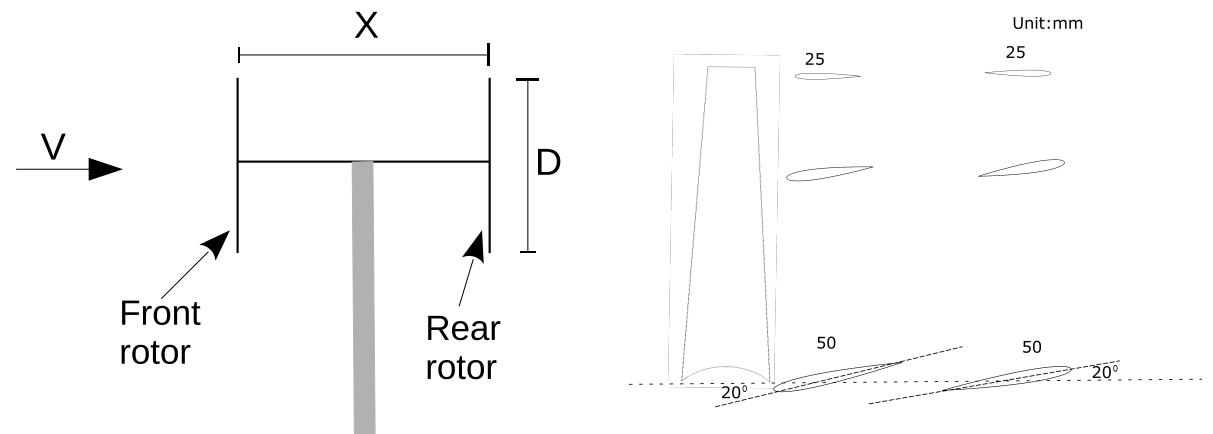

Figure 1: Schematic description of the dual-rotor turbine (a) [16] and blade profile at different stations $(b)$

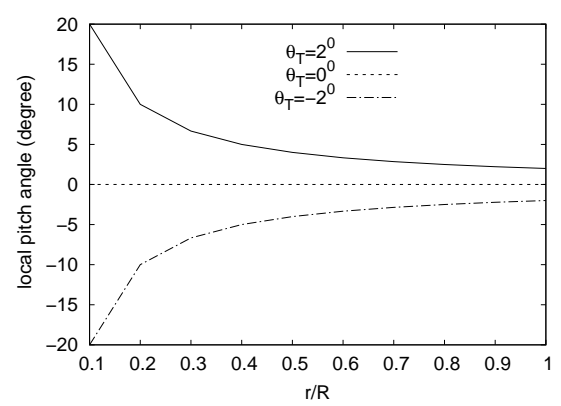

(a)

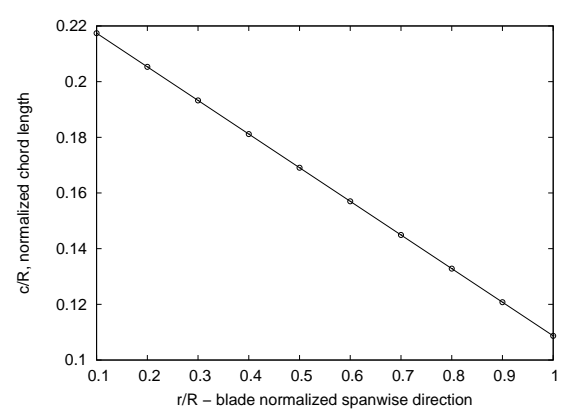

(b)

Figure 2: The local pitch angle (a) and chord length (b) distribution of the NACA0012 blades ( $\theta_{T}$ stands for the pitch angle at the blade tip)

$$
\begin{aligned}
& C_{P}=\frac{2 P}{\rho A U_{\infty}^{3}} \\
& C_{T}=\frac{2 T}{\rho A U_{\infty}^{2}}
\end{aligned}
$$

where, $\Omega$ is the rotational speed of rotor, $R$ is the radius of rotor, $U_{\infty}$ is the

95 free stream velocity, $P$ is the power generated by $\operatorname{rotor}(\mathrm{s}), \rho$ is the density of water, $A$ is the rotational area of rotor, which equals to $\pi R^{2}$, and $T$ stands for the thrust of fluid on rotor.

For a dual-rotor MCT, the power and thrust coefficients are;

$$
C_{P}^{\text {dual }}=\frac{2\left(P_{1}+P_{2}\right)}{\rho A U_{\infty}^{3}}
$$

and

$$
C_{T}^{\text {dual }}=\frac{2\left(T_{1}+T_{2}\right)}{\rho A U_{\infty}^{2}}
$$


where the subscript 1 and 2 denotes the front and rear rotors, respectively. The rotational speed of front rotor is denoted as $\Omega_{1}$, and the rotational speed of rear rotor is denoted as $\Omega_{2}$. Then, the TSR of front rotor is defined as;

$$
T S R_{U_{\infty}}^{\text {front }}=\frac{\Omega_{1} R}{U_{\infty}}
$$

For the rear rotor, its TSR is defined as:

$$
T S R_{\bar{U}}^{r e a r}=\frac{\Omega_{2} R}{\bar{U}}
$$

where, $\bar{U}$ is the velocity seen by the rear rotor. It can also be defined with respect to the free stream velocity as;

$$
T S R_{U_{\infty}}^{r e a r}=\frac{\Omega_{2} R}{U_{\infty}}
$$

\section{Numerical methods}

The BEM-Park model is a combination of the BEM theory and Park wake model 19, where the BEM is used to calculate the steady hydrodynamic performance and thrust loading, and the Park wake model is used to determine the mean axial velocity in the wake region. For the detail of the BEM-Park model, please refer to [16]. Here, only the axial velocity deficit, $\Delta U$, is presented as follow:

$$
\Delta U=U_{\infty}\left(1-\sqrt{1-C_{T}^{\text {front }}}\right)\left(\frac{D}{D+2 k X}\right)^{2}
$$

105

where $C_{T}^{\text {front }}$ is the thrust coefficient of the front rotor, $D$ is the diameter of the rotor, $X$ is the rotor spacing, and $k$ is an empirical factor accounting for the spread of the wake and is taken as 0.04 [20].

The ANSYS Fluent software was used for the Reynolds Averaged NavierStokes (RANS) calculations. To reduce the computational cost, only a 120 degree domain as illustrated in Fig. 3 was considered due to the periodicity in the azimuthal direction for the time averaged flow field [21. The inlet is located three diameters (D) upstream of the front rotor, the outlet is 10D downstream of the rear rotor, and the radial extent of the computational domain is 2.5D. A hybrid mesh was used in this study, with tetrahedron mesh used near the hexahedral mesh was used to reduce the mesh size and improve the mesh in the wake region behind the turbine blade. Fig. 3 shows the mesh in different regions within the computational domain. The inlet velocity is $0.6 \mathrm{~m} / \mathrm{s}$ for all cases, and the $T S R$ was obtained by changing the rotational speed of the rotor.

${ }_{120}$ The Reynolds number was $1.35 \times 10^{5}$ for blade chord length at $0.7 R$ with $T S R$ $=5$. The atmosphere pressure condition was specified at the outlet boundary. A free slip wall was specified at the radial edge of the domain, and periodic 
boundaries were specified at the azimuthal sides. The RANS-based SST $K-\omega$ model was used for the fully geometry-resolved steady simulation. References [22, 23, 24] show that SST $K-\omega$ model can better predict flows with adverse pressure gradient, such as airfoils, wings and rotating blades. Although it is based on the Boussinesq assumption as other two-equation turbulence models, Leroux et al. 25. has demonstrated its accuracy for marine turbines of similar geometry. The Multiple reference frame model was used to account for the 130 rotating of blades. Mesh interfaces were used to transfer the flow data between the stationary frame and the rotation frame. Two turbulence intensity levels $(1 \%$ and $15 \%)$ in the free-stream were investigated in the current study.

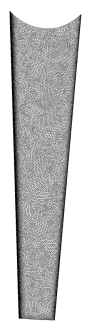

(a)

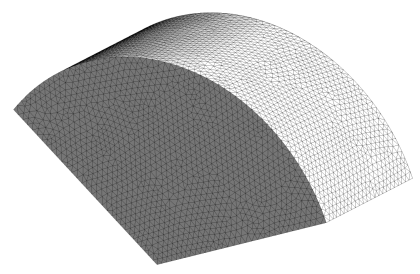

(c)

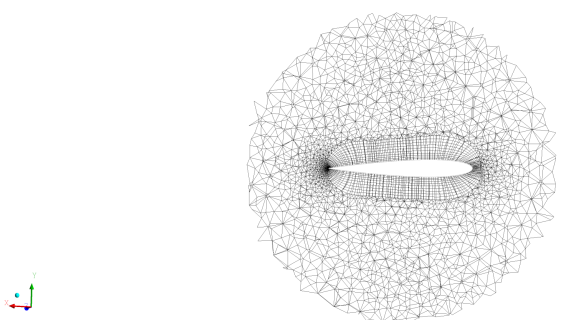

(b)

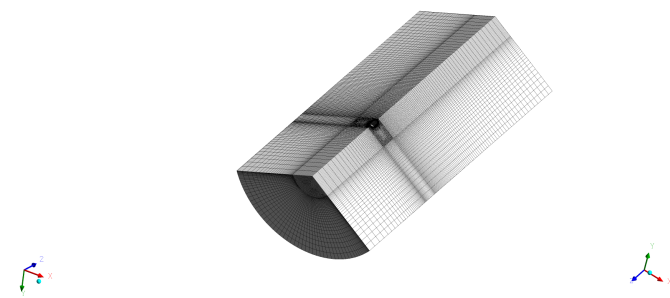

(d)

Figure 3: Mesh details for the turbine

\section{Results and discussion}

\subsection{Grid Convergence}

Fig. 4 presents the pressure coefficient along the blade profile for a singlerotor $\left(\theta_{T}=2^{\circ}\right)$ at $0.9 \mathrm{R}$ and $\mathrm{TSR}=5$. The pressure coefficient is defined as $p=2\left(p-p_{\infty}\right) / \rho U_{\infty}^{2}$. Three different sizes of mesh are used to analysis the grid convergence. Pressure coefficients from 5.4 and 7.1 million meshes have almost identical distribution along the chord length with very mild differences around

$140 \quad 0.1-0.2(\mathrm{x} / \mathrm{c})$ region at the suction surface. The $Y^{+}$value is below 2 for a mesh size of 7.1 million. Thus, for the mesh size of 7.1 million is used for a single-rotor turbine, and 13 million mesh for a dual-rotor turbine. 


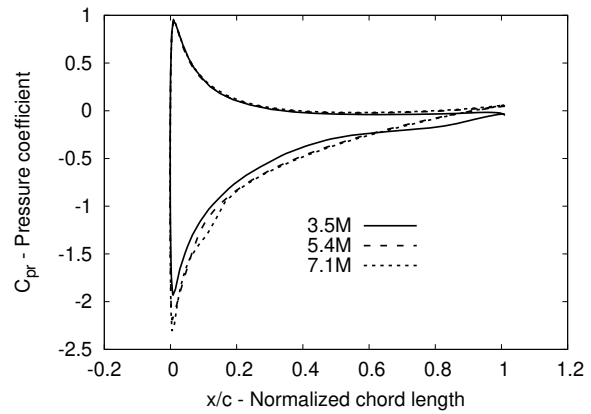

Figure 4: Pressure coefficient of blade profile at $0.9 \mathrm{R}$, single-rotor, TSR $=5 \theta_{T}=2^{\circ}$

4.2. Power and thrust coefficients

4.2.1. Single rotor case

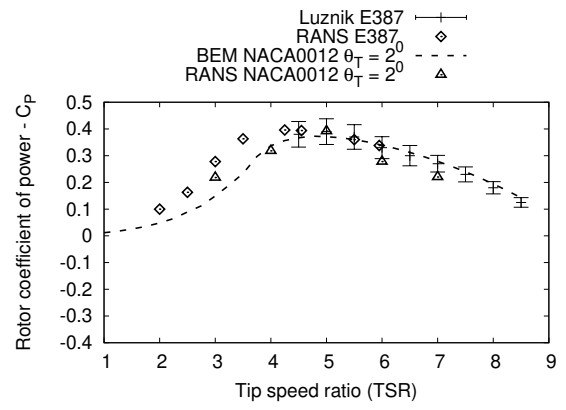

(a)

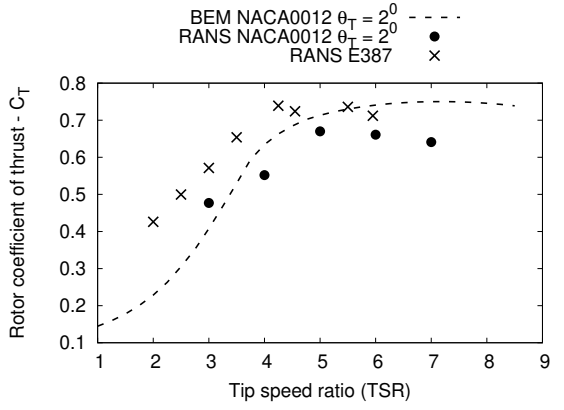

(b)

Figure 5: Isolated front rotor's power (a) and thrust (b) coefficients variations with TSR, (E387 data is from 17 26]) ( $\mathrm{TI}=1 \%$ in RANS)

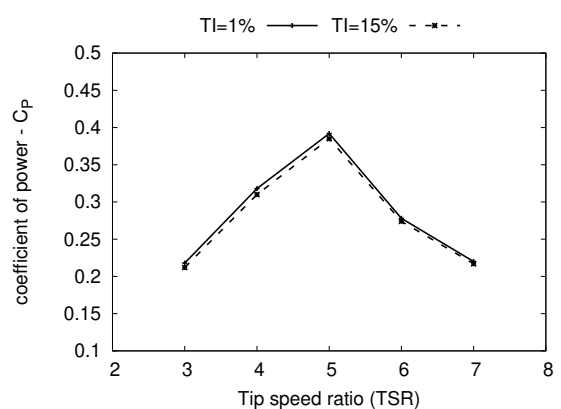

(a)

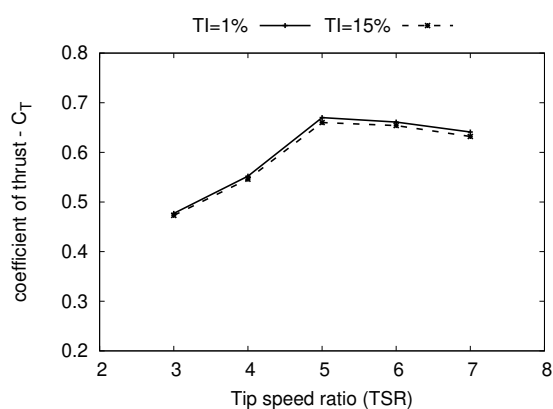

(b)

Figure 6: Isolated front rotor's power (a) and thrust (b) coefficients at different turbulence intensity levels (NACA0012, $\theta_{T}=2^{\circ}$, RANS) 


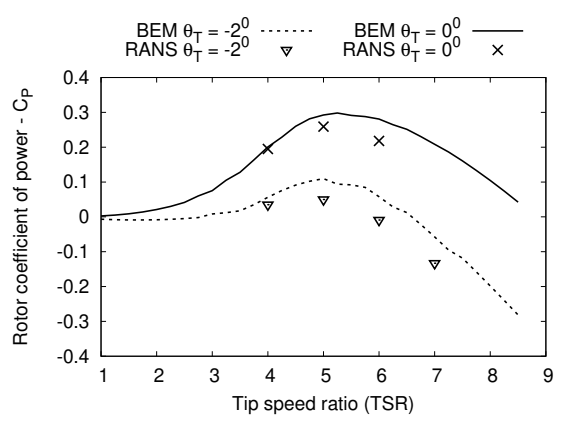

(a)

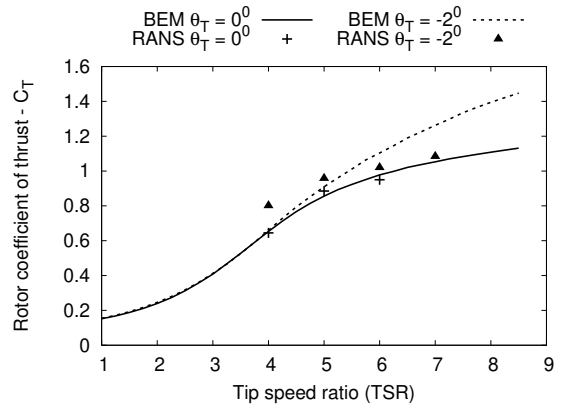

(b)

Figure 7: Single-rotor's coefficient of power (a) and thrust (b) variations with TSR for NACA0012 blades tip-pitched at $\theta_{T}=-2^{\circ}$ and $\theta_{T}=0^{\circ}$ (TI $=1 \%$ in RANS) in water flume tests. Mycek's experimental work 27 showed that a maximum reduction of $13 \%$ in $C_{P}$ was observed when the ambient turbulence intensity increased from a low value of $3 \%$ to a high value of $15 \%$. A $10 \%$ reduction in $C_{P}$ was observed for an increase in TI from $6.8 \%$ to $14.3 \%$ in 30 .

Fig. 7 presents the $C_{P}$ and $C_{T}$ variations with $T S R$ for a NACA0012 singlerotor tip-pitched at $\theta_{T}=\left(-2^{\circ}, 0^{\circ}\right)$. For convenience, the power coefficient of a single-rotor tip-pitch at $\theta_{T}=\left(2^{\circ}, 0^{\circ},-2^{\circ}\right)$ are denoted as $C_{p, \text { single }}^{\text {front }}, C_{p, \text { single }}^{\text {untwist }}$, and $C_{p, \text { single }}^{\text {rear }}$ respectively. The rotor of NACA0012 $\left(\theta_{T}=-2^{\circ}\right)$ has a low positive $C_{P}$ at $T S R=(3-5.5)$ and becomes negative at higher $T S R$. This is as 
expected, since the rear rotor operates in unfavorable pitch angle as illustrated in Fig. 1 (b). The $C_{p, s i n g l e}^{r e a r}$ obtained from RANS is lower than the BEM's value. The maximum positive $C_{p, s i n g l e}^{r e a r}$ gained from BEM is close 0.09 , while its RANS's result is only close to 0.05 . The power coefficient of the E387 rear rotor was found to be highly negative, less than - 0.5 for $T S R=4$ when using RANS

180 or BEM, hence justifying again the use of the symmetric profile NACA0012 for the dual rotor configuration. The negative pitch angle and high TSR also yields a $C_{T}$ much larger than one as seen in Fig. $7 \mathrm{~b}$, which implies a turbulent wake behind the rear rotor. It should be noted that at turbulent wake the conventional momentum thrust expression should be empirically corrected as done in the current BEM code as well as expressions for post stall hydrodynamics of blade profiles 16, 31. This could have contributed to the difference between the BEM and CFD-RANS, where RANS may under-predict post stall hydrodynamic performance.

\subsubsection{Dual rotor case}

The power coefficients of front and rear rotor of a dual-rotor turbine are denoted as $C_{p, \text { dual }}^{\text {front }}$ and $C_{p, d u a l}^{\text {rear }}$, respectively. The challenging part is to specify the rotational speed for the rear rotor $\left(\Omega_{2}\right)$ as $\bar{U}$ is unknown before calculation. Although our previous work [16] can be used to derive the variation of $\Omega_{2} / \Omega_{1}$ as a function of $T S R_{\infty}^{\text {front }}$ (TSR of the front rotor) using the Park model, trial and error method is needed to specify the $\Omega_{2}$ for a fixed $\Omega_{1}$ to account for inaccuracy in the Park model as discussed later. Here, we present the results of the dual-rotor turbine operating with a axial distance of $4 \mathrm{D}$. In the dual-rotor RANS simulation, we let the front rotor operate with a fixed $\Omega_{1}$ and a variable $\Omega_{2}$ for the rear rotor.

200 The power and thrust coefficients of the rear rotor of a dual-rotor MCT are presented in Fig. 8. Two free-stream turbulence intensities are considered because the rear rotor of a dual-rotor turbine operates in the wake region of the front rotor, where velocity recovery rate is strongly influenced by the turbulence levels. The first obvious observation is that the power and thrust coefficients of the rear rotor operating in the wake region of the front rotor are much lower than an isolated rotor's counterparts. The $C_{P, \text { dual }}^{\text {rear }}$ is lower than $C_{P, \text { single }}^{\text {rear }}$ is mainly due to the reduced velocity in the wake region of front rotor. The RANS results show that the maximum $C_{P, d u a l}^{\text {rear }}$ is close to zero at TSR $=2.5$ with $T I=1 \%$, which increases to 0.02 at TSR $=3.2$ with $T I=15 \%$. However, the maximum ${ }_{210} C_{P, \text { dual }}^{\text {rear }}$ obtained from BEM-Park model is 0.04, which is higher than its RANS counterparts [16. The optimum TSR of the rear rotor with $T I=15 \%$ is around 3 , while it is mildly high at about 3.6 in the BEM result [16]. For the same TSRs, the rear rotor always extracts more power at the high turbulence level $(T I=15 \%)$ than the low turbulence level $(T I=1 \%)$. Similar trend is observed for the thrust coefficient of the rear rotor, as presented in Fig. 8 (b).

Fig. 9 shows the total power performance of the dual-rotor MCT, with the single-rotor results for comparison. When the front rotor operates at $T S R=5$, and rear rotor operates at $T S R=3.2$ with $T I=15 \%$, the $C_{P}$ obtained from 


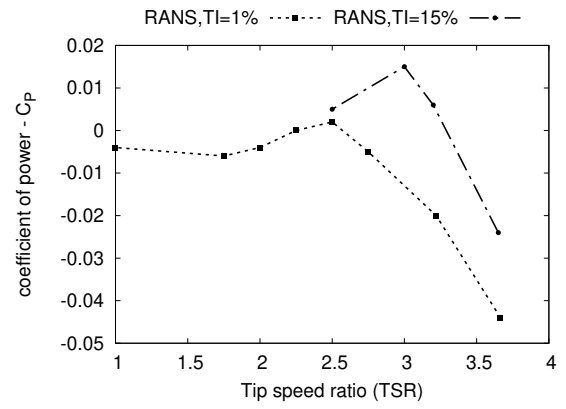

(a)

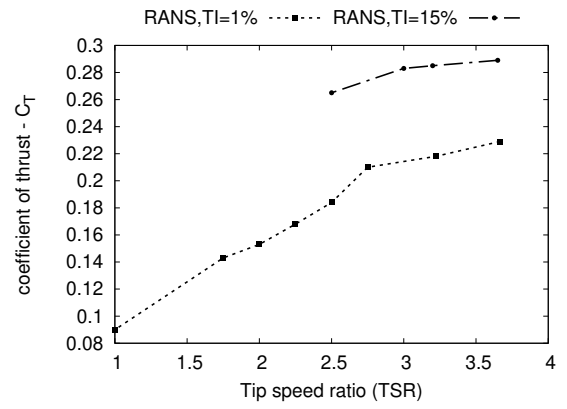

(b)

Figure 8: Power (a) and thrust (b) coefficients of the rear rotor of a dual-rotor $(\mathrm{X}=4 \mathrm{D})$ with variable $T S R_{U_{\infty}}^{\text {rear }}$ and a fixed $T S R_{U_{\infty}}^{\text {front }}=5$

RANS results matches that of the BEM-Park model. The overall $C_{P}$ of the dual-rotor MCT is $5 \%$ higher than its single-rotor counterpart based on the RANS and BEM-Park model results. The RANS results show that the difference between $C_{p, \text { single }}^{\text {front }}$ and $C_{p, \text { dual }}^{\text {front }}$ is within $0.5 \%$ when the front rotor operates at fixed $T S R$, and the rear rotor operates at variable TSRs. This means the rear rotor has little effect on the front rotor for a dual-rotor MCT with a distance $X=4 D$. This numerical observation is consistent with similar experimental work done by Mycek [32, who studied the single-rotor MCT arrays in tandem distribution, and the rotors were pitched in the same direction.

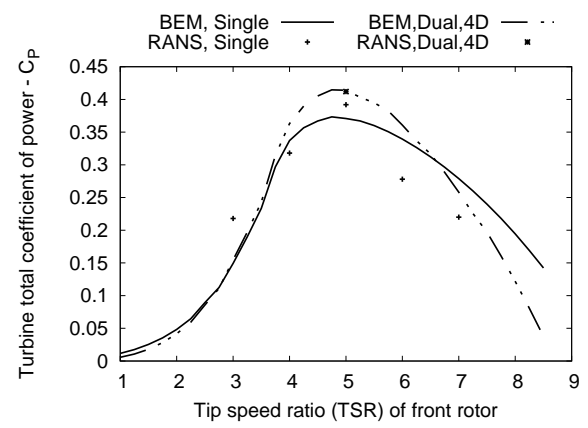

Figure 9: Dual-rotor's total power coefficient versus the front rotor's TSR (In RANS, $T S R_{U_{\infty}}^{\text {front }}=5$, and $T S R_{U_{\infty}}^{\text {rear }}=3.2$ for the rear rotor ), compared to single-rotor tip-pitched at $\theta_{T}=2^{\circ}$. (BEM data is from [16])

\subsection{Park wake model evaluation}

The previous section has shown good agreement between RANS and BEM 230 for the single rotor pitched at $\theta_{T}=2^{\circ}$ and also good agreement for the dualrotor at the high $\mathrm{TI}=15 \%$, but not $1 \%$. Obviously, the Park model may have difficulties predicting the velocity deficit in the wake for low turbulence intensity 
and deserves further investigation. The mean axial velocity behind the front rotor is a key factor for the power performance of the rear rotor. The mean axial velocity in the wake region obtained from RANS is area averaged and compared to the velocity deficit derived from the Park wake model. In the RANS calculation, the area averaged axial mean velocity in the wake region, $\bar{U}$, is defined as:

$$
\bar{U}=\frac{\iint_{A(r)} U(x, y, z) d y d z}{A(r)}
$$

where $U$ is the mean axial velocity, $A(r)$ is a sectional area of wake region at a given axial distance $(\mathrm{X} / \mathrm{D})$, and the sectional area is parallel to the rotational plane. Fig. 10a illustrates a sectional wake area after a single-rotor at an axial distance $\mathrm{X}$.

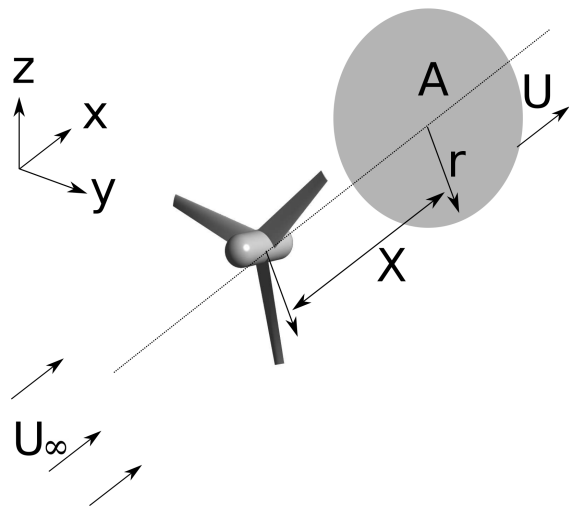

(a)

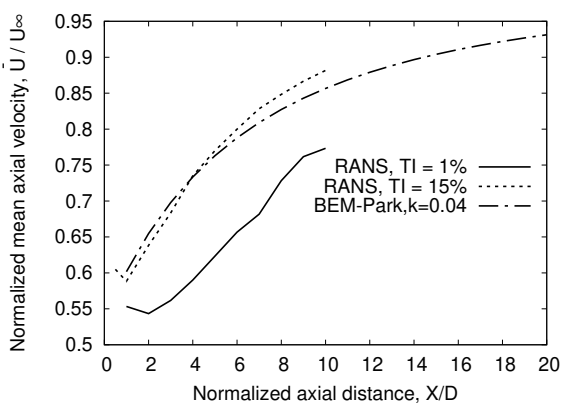

(b)

Figure 10: Schematic sectional wake region at axial distance $\mathrm{X}$ (a), and normalized mean axial velocity downstream of a single-rotor tip-pitched at $\theta_{T}=2^{\circ}, \mathrm{TSR}=5(\mathrm{~b})$

Fig. 10b shows the mean axial velocity as a function of the non-dimensional axial distance after a single-rotor $\left(\theta_{T}=2^{\circ}\right)$ which operates at TSR 5 . Two ence intensity levels are available from the CFD results, namely $1 \%$ and $15 \%$. One should note, these turbulent intensities correspond to the low and high levels of turbulence intensity commonly used in a water tank tests and field sites of tidal turbines [27. The recommended value of $\mathrm{k}$ is 0.04 as used in our previous work [16. For RANS results, the axial mean velocity grows as the axial distance increases except in the very near wake of $1-2 \mathrm{D}$, where a decrease of $\bar{U}$ is observed. However, a monotonic increase of $\bar{U}$ is observed in the Park model. There is a better agreement between BEM-Park model and RANS at the high turbulence intensity level $(\mathrm{TI}=15 \%)$ than the low turbulence level(TI=1\%). In the near wake region $(X / D<4)$, the normalized axial mean velocity obtained from 255 BEM-Park Model is mildly higher than its RANS's counterpart at TI=15\%. However, In the far wake region $(X / D>4)$, the normalized axial mean velocity 
obtained from BEM-Park Model is mildly lower than its RANS's counterpart at $\mathrm{TI}=15 \%$. However, at $\mathrm{TI}=1 \%$, the RANS model predicts a much lower velocity than the Park model, explaining the much better agreement between the RANS and BEM-Park predicts for the dual-rotor with $\mathrm{TI}=15 \%$.

The value of the empirical wake expansion rate, $k$, is an important parameter for the velocity deficit calculation in the Park model. The value of $k=0.04$ is recommended in the wind farm, while this value may not appropriate for the MCT. For the marine environment, a relation of $k=0.4 T I$ was recommended 265 by Pena 33 and Goccmen 34 to account for the turbulence intensity effect. However, this relationship is not accurate for our cases. Pyakurel [35] proposed a calibaration of $\mathrm{k}$ using the mean velocity along the center line. The calibrated $k$ values are $0.0325(\mathrm{TI}=3 \%), 0.0477(\mathrm{TI}=6 \%)$, and $0.0679(\mathrm{TI}=9 \%)$. However, this correction is less accurate because the wake shape behind an isolated marine current turbine normally forms a Gaussian shape distribution, and the axial mean velocity is minimum at the center line. Calibration based on area-averaged axial mean velocity will result in better approximation. Based on the $C_{T}$ of front rotor and area-averaged axial mean velocity from RANS results, the calibrated value of $\mathrm{k}$ is $0.017(\mathrm{TI}=15 \%)$ using the least square fitting [36].

\subsection{Fluid visualization}

\subsubsection{Streamlines}

The streamline is a useful tool for locating flow separation and vortex region. A sectional streamline around the blade station gives more insight on local flow conditions. Fig. 11 shows the side view of streamlines of both single and dual280 rotors at TSR 5. The inconsistency of streamline at the mesh interface is due to different meshing types used in the outer cell zone and inner cell zone. The first striking observation is the existence of a vortex in the wake region in all three cases. The vortex size is about $0.5 \mathrm{D}$ for a single-rotor tip-pitched at $\theta_{T}=2^{\circ}$, while a much larger size of vortex $(6 \mathrm{D})$ is observed for a single-rotor tip-pitched at $\theta_{T}=-2^{\circ}$. For a dual-rotor with front rotor tip-pitched at $\theta_{T}=2^{\circ}$, the size of the vortex downstream of the front and rear rotors are almost the same as that of the single-rotor cases, although the shape of the vortex downstream of rear rotor (Fig. 11 (c) ) is much more squeezed compared to that of a single-rotor tip-pitched at $\theta_{T}=-2^{\circ}$. An interesting observation is that a new vortex is formulated in front of the rear rotor.

Fig. 12 shows sectional streamlines of single-rotor turbines tip-pitched at $\theta_{T}=\left(2^{\circ},-2^{\circ}\right)$ operating at $\mathrm{TSR}=5$. A laminar separation bubble (LSB) is observed near the leading edge of the profile when $r / R$ is below 0.5 , and the LSB moves towards the leading edge as $r / R$ decreases. The LSB is near the 295 inner board of blade and thickens the boundary layer, thus contributes to the decrease of profile hydrodynamic efficiency, $C_{L} / C_{D}$. It should bear in mind that the angle of attack is relative to incoming free velocity vector seen by the blade profile and not the local slope of the streamline just in front of the blade profile, although they are not far from each other. To provide better estimate of the AOA from the CFD, several methods were allowed in the literature [37, 38, 39]. 
(a)

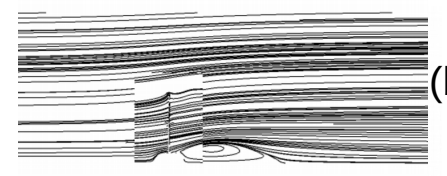

(b)

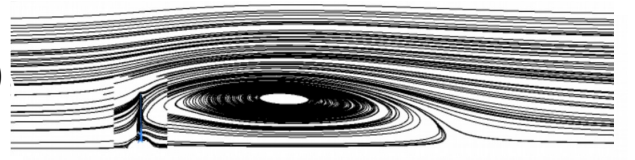

(c)

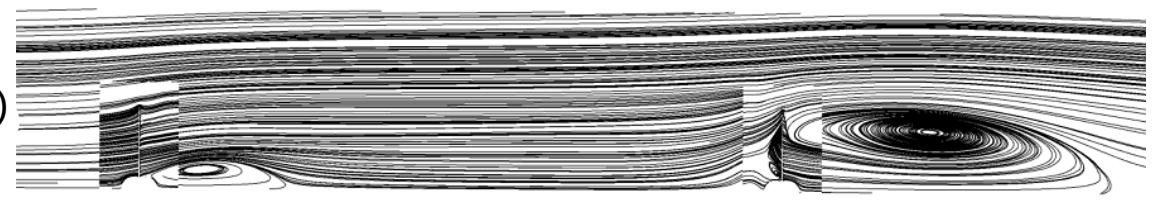

Figure 11: Side view streamline of single and dual-rotors at TSR 5, TI=1\% (RANS)

This can be important for a coupled CFD-BEM method. For this study, we just note the good resemblance between the CFD and BEM's results.

Fig. 13 presents the local streamlines of rear rotor of a dual-rotor with fixed TSR of front rotor and variable $T S R_{\bar{U}}$ of rear rotor. Here, $\bar{U}$ is the area averaged mean axial velocity after a single-rotor tip-pitched at $\theta_{T}=2^{\circ}$ at $\mathrm{X}=4 \mathrm{D}$. At low $T S R_{\bar{U}}(2.91,3.75)$, there are large flow separation from the rear surface of the blade, while no flow separation is observed at high $T S R_{\bar{U}}$, such as $T S R_{\bar{U}}=6$.

Fig. 14 presents the 3D streamline of single and dual-rotors. A nice screwlike vortex is seen behind the single-rotor at $\theta_{T}=2^{\circ}$ in Fig. 14 (a), pointing 310 to its high hydrodynamic efficiency. A large separated wake is seen behind the single-rotor of $\theta_{T}=-2^{\circ}$, showing its low hydrodynamic efficiency. Illustration of the vortical wake behind the front rotor and how it affects the wake behind the rear rotor is shown in Figs 14(c) \& (d). The vortical wake shedded by the front rotor is seen to reduce the wake behind the rear rotor compared to the single-rotor wake and thus has the potential actually to mildly increase the rear rotor hydrodynamic efficiency, explaining the high $C_{P}$ seen in Fig. 9 (b).

\subsubsection{Velocity contour and turbulence intensity}

The evolution of the axial mean velocity in the wake region was presented in Fig. 10. For better fluid visualization, velocity contours are illustrated in order 320 to enable better understanding of the potential in the dual-rotor configuration. Figs. 15, 16, 17, and 18 present velocity contours of a single-rotor tip-pitched at $\theta_{T}=2^{\circ}, \theta_{T}=-2^{\circ}$ and a dual-rotor with rotor spacing of $4 \mathrm{D}$. The wake regions are slightly larger than the rotor diameter in the radial direction. Meanwhile, a much longer low velocity region is observed for a single-rotor tip-pitched at

${ }_{325} \theta_{T}=-2^{\circ}$ compared with its $\theta_{T}=2^{\circ}$ counterpart. This is expected by the low hydrodynamic performance of the rotor at $\theta_{T}=-2^{\circ}$ that also yielded a high $C_{T}$ which is an indicator to a turbulent wake. For a single-rotor tip-pitched at $\theta_{T}=2^{\circ}$, the wake shape is axisymmetric, while a non-axisymmetric wake is observed for a single-rotor tip-pitched at $\theta_{T}=-2^{\circ}$ and a dual-rotor with rotor 


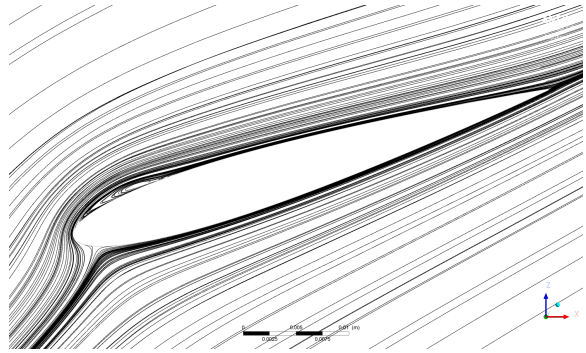

(a) $0.2 \mathrm{R}$

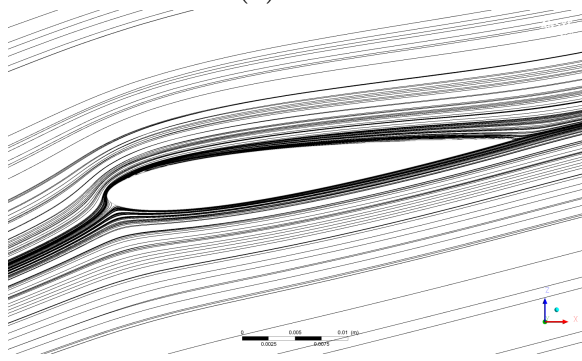

(c) $0.5 \mathrm{R}$

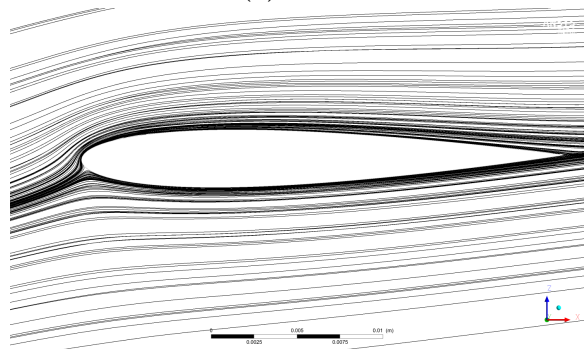

(e) $0.9 \mathrm{R}$

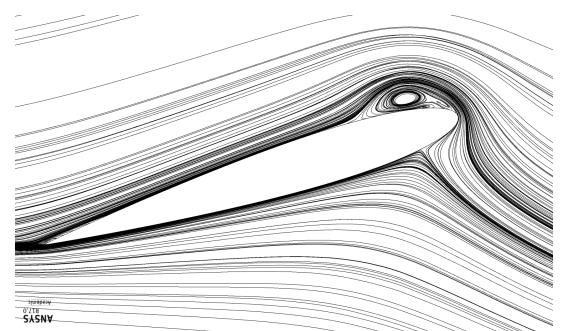

(b) $0.2 \mathrm{R}$

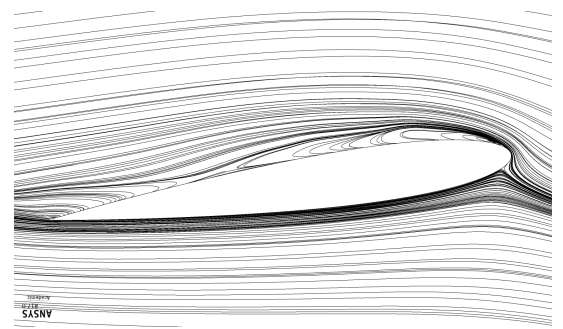

(d) $0.5 \mathrm{R}$

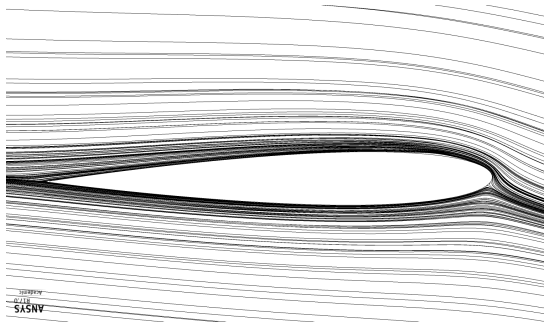

(f) $0.9 \mathrm{R}$

Figure 12: Local streamlines of single-rotor turbines at TSR 5, TI $=1 \%, \theta_{T}=2^{\circ}$ (left) and $\theta_{T}=-2^{\circ}$ (right)

spacing of $4 \mathrm{D}$.

Fig. 19 presents side view of the turbulence intensity of a single and dualrotors operating at TSR 5 with ambient turbulence intensity of 1\%. Although our model is a two-equation turbulence model, the $k-\omega$ SST RANS model was seen to produce reasonably accurate wake description behind the MCT, including the turbulence intensity [25].

Similarly as the side view of velocity contour, for a single-rotor tip-pitched at $\theta_{T}=-2^{\circ}$, the area of high turbulence intensity is much larger than its $\theta_{T}=2^{\circ}$ counterpart. For a single-rotor tip-pitched at $\theta_{T}=-2^{\circ}$, a high turbulence intensity region (such as $T I>0.28$ ) extends to $5 \mathrm{D}$ downstream after the rotor and $0.6 \mathrm{D}$ in radial direction, while the high turbulence intensity region is mainly constrained near the hub for a single-rotor tip-pitched at $\theta_{T}=2^{\circ}$. For a dual- 


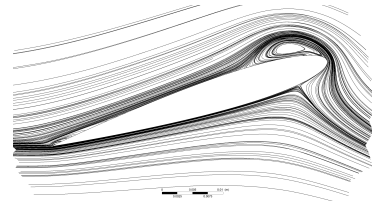

(a) $0.2 \mathrm{R}, T S R_{\bar{U}}=2.91$

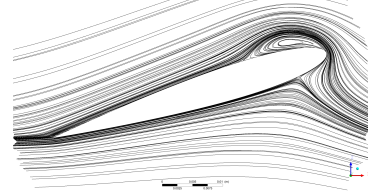

(d) $0.2 \mathrm{R}, T S R_{\bar{U}}=3.75$

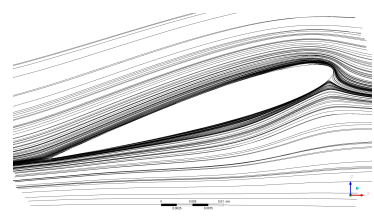

(g) $0.2 \mathrm{R}, T S R_{\bar{U}}=6$

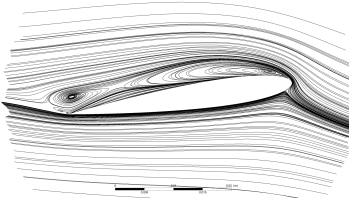

(b) $0.5 \mathrm{R}, T S R_{\bar{U}}=2.91$

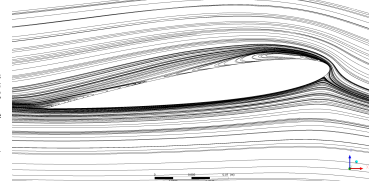

(e) $0.5 \mathrm{R}, T S R_{\bar{U}}=3.75$

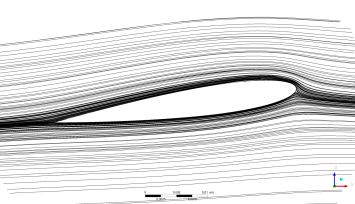

(h) $0.5 \mathrm{R}, T S R_{\bar{U}}=6$

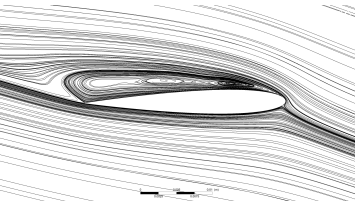

(c) $0.9 \mathrm{R}, T S R_{\bar{U}}=2.91$

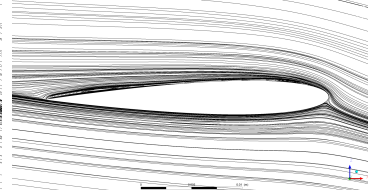

(f) $0.9 \mathrm{R}, T S R_{\bar{U}}=3.75$

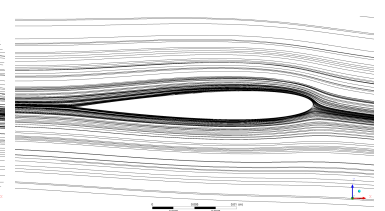

(i) $0.9 \mathrm{R}, T S R_{\bar{U}}=6$

Figure 13: Local streamline of the rear rotor of dual-rotor $(\mathrm{X}=4 \mathrm{D})$ with $T S R^{\text {front }}=5$ and variable $T S R^{\text {rear }}(\mathrm{TI}=1 \%$, RANS, $\bar{U}$ is the mean axial velocity downstream a single-rotor tip-pitched $\theta_{T}=2^{\circ}$ )

rotor with $\mathrm{X}=4 \mathrm{D}$, an interesting observation is that a turbulence intensity region is developed in front of rear rotor. 

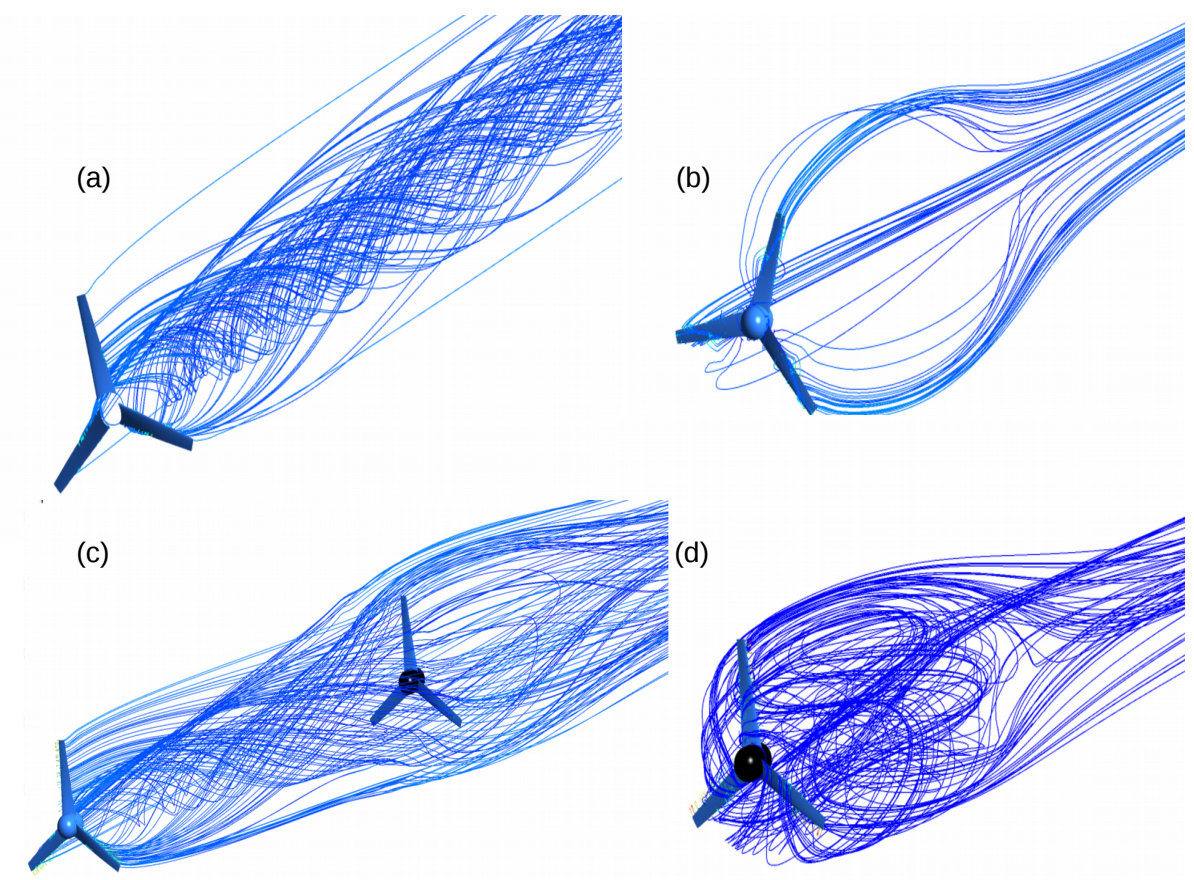

Figure 14: 3D streamlines behind single and dual-rotors $(\mathrm{X}=4 \mathrm{D})$ at TSR 5, (a) single-rotor $\theta_{T}=2^{\circ}$, (b) single-rotor $\theta_{T}=-2^{\circ}$, (c) dual-rotor, streamline starts from front rotor, (d) dual-rotor, streamline starts from rear rotor
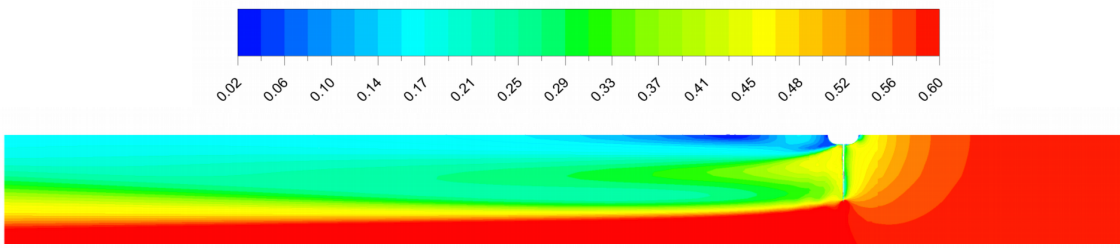

(a)

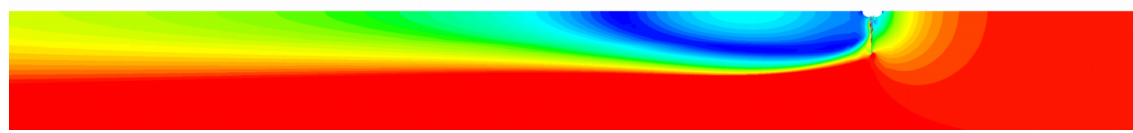

(b)

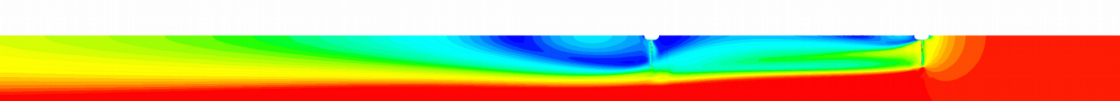

Figure 15: Side view velocity contour of single and dual-rotors, (a) $\theta_{T}=2^{\circ}$, (b) $\theta_{T}=-2^{\circ}$, and (c)dual rotor with $\mathrm{X}=4 \mathrm{D}\left(T S R^{\text {front }}=5, T S R^{\text {rear }}=3.5\right)($ inlet at right side) 


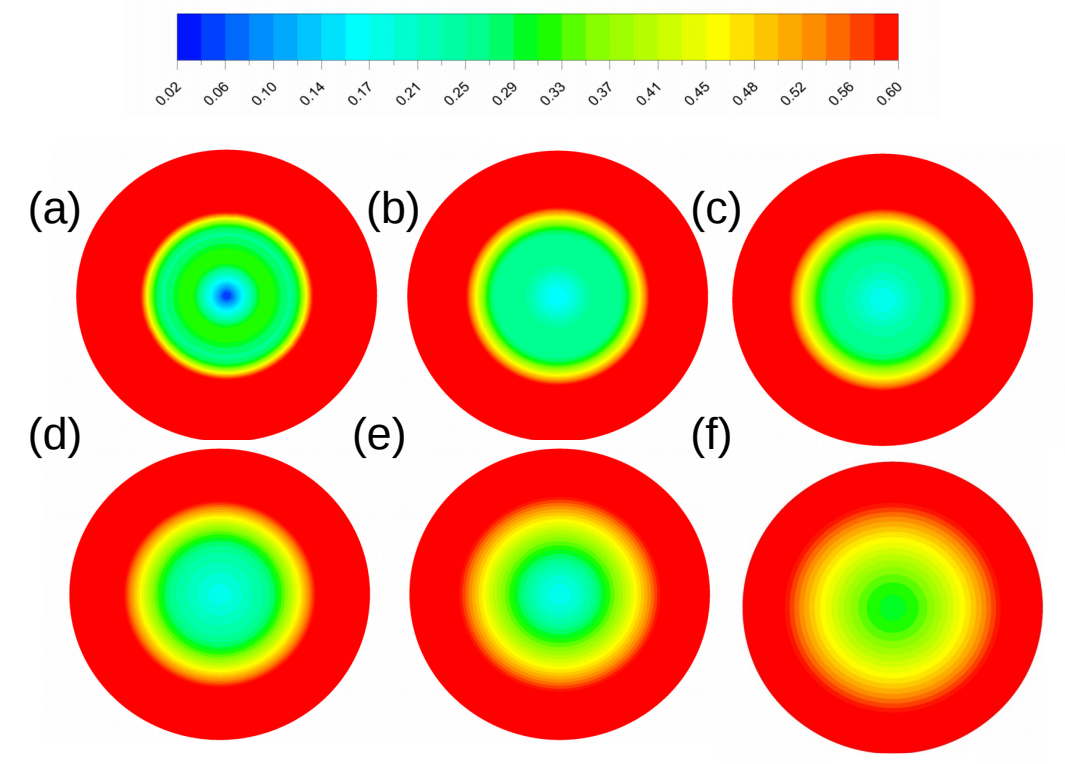

Figure 16: Front view of the velocity contours behind isolated front rotor $\left(\theta_{T}=2^{\circ}\right)$ at TSR 5 , (a) $1 \mathrm{D}$, (b) 3D, (c) 4D, (d) 5D, (e) 7D, (f) $10 \mathrm{D}$

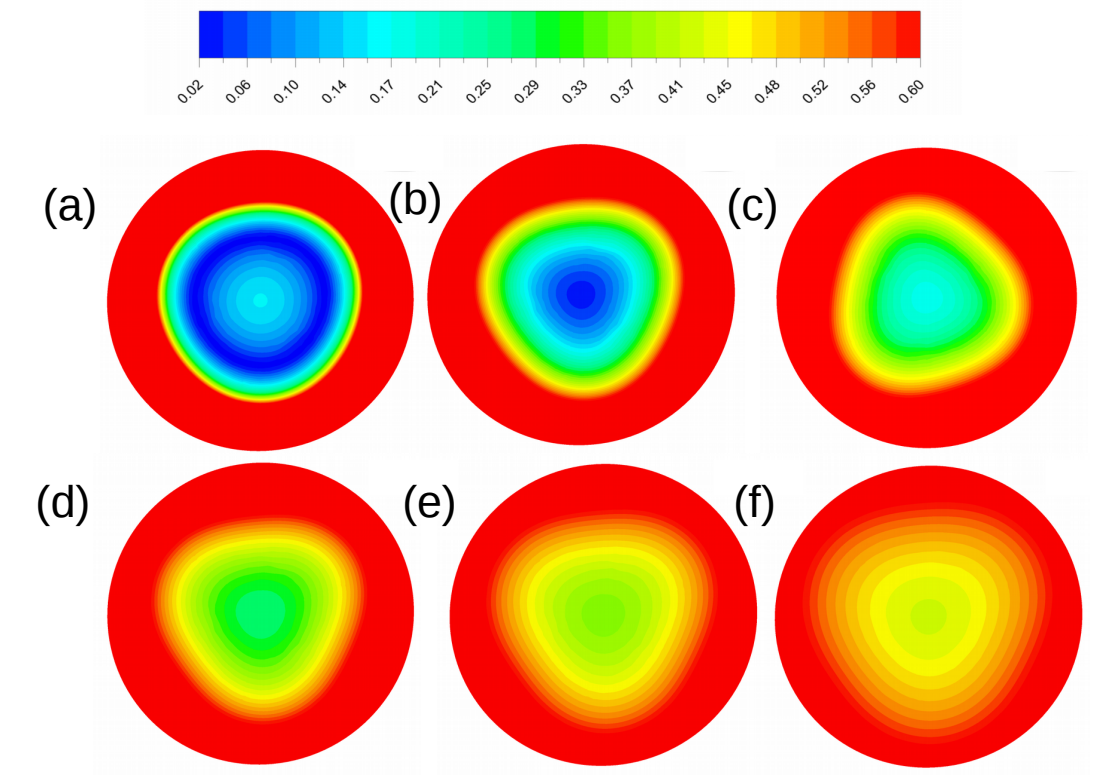

Figure 17: Front view of the velocity contours behind isolated rear rotor $\left(\theta_{T}=-2^{\circ}\right)$ at TSR 5, (a) 1D, (b) 3D, (c) 4D, (d) 5D, (e) 7D, (f) 10D 


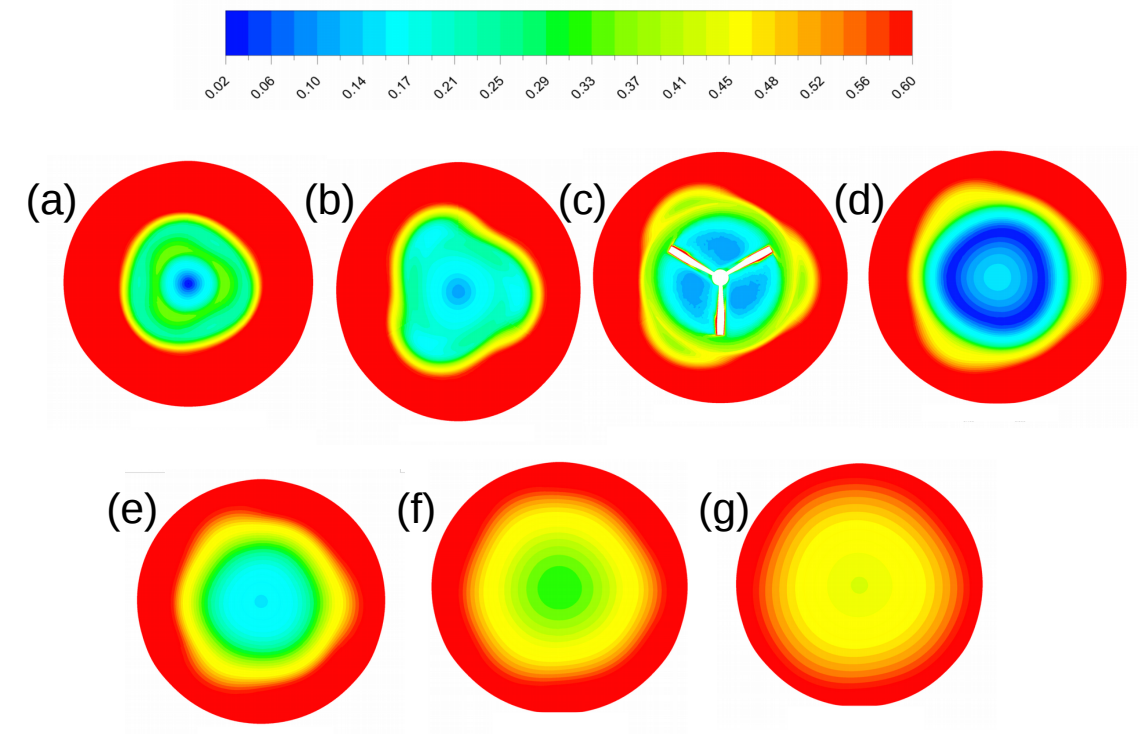

Figure 18: Front view of the velocity contour of dual-rotor with $\mathrm{X}=4 \mathrm{D}$ at TSR 5 ,(a) 1D, (b) $3 \mathrm{D}$, (c) $4 \mathrm{D}$, (d) $5 \mathrm{D}$, (e) $7 \mathrm{D}$, (f) $10 \mathrm{D}$, (g) $14 \mathrm{D}$ ( front rotor, $\theta_{T}=2^{\circ}$, origin is located at the axis center of front rotor )

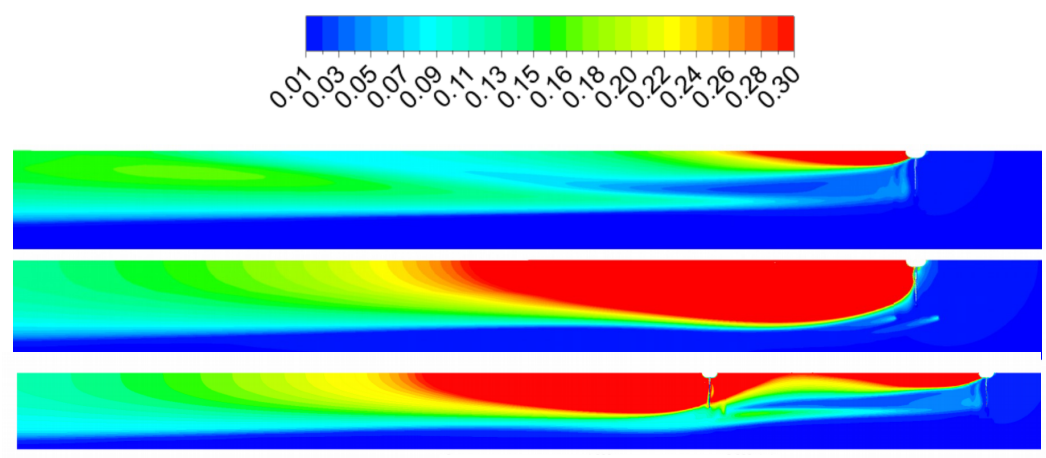

(a)

(b)

(c)

Figure 19: Side view turbulence intensity of single and dual-rotors at TSR 5, (a) isolated front rotor, (b) isolated rear rotor, (c) dual rotor at $\mathrm{X}=4 \mathrm{D}(\mathrm{TI}=1 \%)$ 


\section{Conclusion}

345 a rotational speed control but with no pitch and yaw controls. The turbine is aligned for a rectilinear tidal current, thus the rotors are oppositely pitched. The RANS-based SST $k-\omega$ turbulence model was used to simulate both singlerotor and dual-rotor MCTs. Power and thrust coefficients, mean axial velocities model. The CFD results provided more details, such as velocity contours, and streamlines.

For a single-rotor $\mathrm{MCT}$, there was a good agreement for $C_{P}$ between $\mathrm{BEM}$ and CFD results, except when the rotor operated at high TSRs or having a 355 turbulent wake state with negatively pitched blades. For a dual-rotor MCT, larger discrepancies were observed compared with single-rotor cases. The $C_{P}$ of rear rotor obtained from BEM-Park model was higher than its CFD RANS counterpart. Nevertheless, the CFD results shows that the BEM-Park model provides reasonable accuracy for the total performance of the dual-rotor MCT when in presence of high turbulence intensity. A gain of $5 \%$ and $4 \%$ in the overall $C_{P}$ was recorded as relative to the $C_{P}$ of single-rotor NACA0012 and E387, respectively. The free-stream TI is a key parameter for the $C_{P}$ of rear rotor. A higher TI is preferred for a faster recovery of velocity in the wake region.

365 This analysis shows that there is ground to improve low-order models to better account the incoming TI. It also assumes the rotational plane of rotor is orthogonal to the incoming tidal current. Hence, this study did not account for negative effects from yawed inflow, free surface waves and sea bed. The unsteady RANS simulation can also be used to improve the prediction of performance and

370 load on rear rotor which operates in turbulent windmill state and experiences larger fluctuations during one periodic cycle. Experimental work on dual-rotor case is also recommended for further investigation.

\section{Acknowledgement}

The first author would like to thank the Queen Mary China Scholarship 375 Council Co-funded Scholarships. This research utilised Queen Mary's Apocrita HPC facility, supported by QMUL Research-IT. http://doi.org/10.5281/zenodo.438045

\section{References}

[1] K.-W. Ng, W.-H. Lam, K.-C. Ng, 2002-2012: 10 years of research progress in horizontal-axis marine current turbines, Energies 6 (3) (2013) 1497-1526.

380

[2] Y. J. Gu, Y. G. Lin, Q. K. Xu, H. W. Liu, W. Li, Blade-pitch system for tidal current turbines with reduced variation pitch control strategy based on tidal current velocity preview, Renewable Energy 115. 
[3] B. G. Newman, Actuator-disc theory for vertical-axis wind turbines, Journal of Wind Engineering and Industrial Aerodynamics (1983) 347-355.

[4] B. Newman, Multiple actuator-disc theory for wind turbines, Journal of Wind Engineering and Industrial Aerodynamics 24 (3) (1986) 215-225.

[5] H. Sundararaju, K. H. Lo, R. Metcalfe, S. S. Wang, Aerodynamics and cfd analysis of equal size dual-rotor wind turbine, Journal of Renewable and Sustainable Energy 9 (4) (2017) 043305.

[6] W. Z. Shen, V. A. K. Zakkam, J. N. Srensen, K. Appa, Analysis of counterrotating wind turbines, in: Journal of Physics Conference Series, 2007, p. 012003.

[7] A. Vaselbehagh, C. L. Archer, Wind farms with counter-rotating wind turbines, Sustainable Energy Technologies and Assessments 24 (2017) 19-30.

[8] S. Lee, E. Son, S. Lee, Velocity interference in the rear rotor of a counterrotating wind turbine, Renewable Energy 54 (2013) 235-240.

[9] S. N. Jung, T. No, K. Ryu, Aerodynamic performance prediction of a 30kw counter-rotating wind turbine system, Renewable Energy 30 (5) (2005) 631-644.

[10] W. Cho, K. Lee, I. Choy, J. Back, Development and experimental verification of counter-rotating dual rotor/dual generator wind turbine: Generating, yawing and furling, Renewable Energy 114 (2017) 644-654.

[11] Z. Wang, A. Ozbay, W. Tian, H. Hu, An experimental study on the aerodynamic performances and wake characteristics of an innovative dual-rotor wind turbine, Energy 147 (2018) 94-109.

[12] B. Hwang, S. Lee, S. Lee, Optimization of a counter-rotating wind turbine using the blade element and momentum theory, Journal of Renewable and Sustainable Energy 5 (5) (2013) 052013.

[13] J. Clarke, G. Connor, A. Grant, C. Johnstone, Design and testing of a contra-rotating tidal current turbine, Proceedings of the Institution of Mechanical Engineers, Part A: Journal of Power and Energy 221 (2) (2007) 171-179.

[14] B. Huang, Y. Nakanishi, T. Kanemoto, Numerical and experimental analysis of a counter-rotating type horizontal-axis tidal turbine, Journal of Mechanical Science and Technology 30 (2) (2016) 499-505.

[15] B. Huang, G. J. Zhu, T. Kanemoto, Design and performance enhancement of a bi-directional counter-rotating type horizontal axis tidal turbine, Ocean Engineering 128 (2016) 116-123. 
[16] E. Avital, K. Ai, N. Venkatesan, A. Samad, T. Korakianitis, Hydrodynamic assessment of a dual-rotor horizontal axis marine current turbine, International Journal of Engineering and Technology (UAE) (2018) 455-459.

[17] L. Luznik, K. A. Flack, E. E. Lust, K. Taylor, The effect of surface waves on the performance characteristics of a model tidal turbine, Renewable energy 58 (2013) 108-114.

${ }_{425}$ [18] A. Rosen, Wind turbines lecture notes, Technion press, 1987.

[19] N. Jensen, A note on wind generator interaction, Risø National Laboratory, 1983.

[20] J. R. Marden, S. D. Ruben, L. Y. Pao, A model-free approach to wind farm control using game theoretic methods, IEEE Transactions on Control Systems Technology 21 (4) (2013) 1207-1214.

[21] Fluent, Ansys fluent theory guide, Ansys Inc.

[22] J. Mcnaughton, Turbulence modelling in the near-field of an axial flow tidal turbine in code_saturne, Ph.D. thesis, School of Mechanical, Aerospace and Civil Engineering (2013).

${ }_{435}$ [23] M. Rahimian, J. M. Walker, I. Penesis, Numerical assessment of a horizontal axis marine current turbine performance, International Journal of Marine Energy 20 (2017) 151-164.

[24] T. Karthikeyan, E. Avital, N. Venkatesan, A. Samad, Design and analysis of a marine current turbine, in: ASME 2017 Gas Turbine India Conference, American Society of Mechanical Engineers, 2017, pp. V001T02A014V001T02A014.

[25] T. Leroux, N. Osbourne, D. Groulx, Numerical study into horizontal tidal turbine wake velocity deficit: Quasi-steady state and transient approaches, Ocean Engineering 181 (2019) 240-251.

${ }_{445}$ [26] A. K, E. Avital, S. X, S. A, V. N, The surface curvature effect on performance of a laboratory scale tidal turbine, in: IAENG World Congress on Engineering WCE, IEEE, 2018, pp. 571-575.

[27] P. Mycek, B. Gaurier, G. Germain, G. Pinon, E. Rivoalen, Experimental study of the turbulence intensity effects on marine current turbines behaviour. part i: One single turbine, Renewable Energy 66 (2014) 729-746.

[28] E. E. Lust, K. A. Flack, L. Luznik, Survey of the near wake of an axial-flow hydrokinetic turbine in quiescent conditions, Renewable Energy 129 (2018) 92-101. 
[29] K. Ai, E. Avital, T. Korakianitis, A. Samad, N. Venkatesan, Surface wave effect on marine current turbine, modelling and analysis, in: Mechanical and Aerospace Engineering (ICMAE), 2016 7th International Conference on, IEEE, 2016, pp. 180-184.

[30] T. Blackmore, L. E. Myers, A. B. S. Bahaj, Effects of turbulence on tidal turbines: Implications to performance, blade loads, and condition monitoring, International Journal of Marine Energy 14 (2016) 1-26.

[31] J. Tangler, D. Kocurek, Wind turbine post-stall airfoil performance characteristics guidelines for blade-element momentum methods, in: 43rd AIAA Aerospace Sciences Meeting and Exhibit, 2005, p. 591.

[32] P. Mycek, B. Gaurier, G. Germain, G. Pinon, E. Rivoalen, Experimental study of the turbulence intensity effects on marine current turbines behaviour. part ii: Two interacting turbines, Renewable Energy 68 (2014) 876-892.

[33] A. Peña, P.-E. Réthoré, M. P. van der Laan, On the application of the jensen wake model using a turbulence-dependent wake decay coefficient: the sexbierum case, Wind Energy 19 (4) (2016) 763-776.

[34] T. Göçmen, P. Van der Laan, P.-E. Réthoré, A. P. Diaz, G. C. Larsen, S. Ott, Wind turbine wake models developed at the technical university of denmark: A review, Renewable and Sustainable Energy Reviews 60 (2016) $752-769$.

[35] P. Pyakurel, W. Tian, J. H. VanZwieten, M. Dhanak, Characterization of the mean flow field in the far wake region behind ocean current turbines, Journal of Ocean Engineering and Marine Energy 3 (2) (2017) 113-123.

[36] W. H. Press, S. A. Teukolsky, W. T. Vetterling, B. P. Flannery, Numerical recipes in FORTRAN 90 the art of parallel scientific computing, Vol. 2, Cambridge University Press, 1996.

[37] S. Guntur, N. N. Sørensen, An evaluation of several methods of determining the local angle of attack on wind turbine blades, in: Journal of Physics: Conference Series, Vol. 555, IOP Publishing, 2014, p. 012045.

[38] E. Jost, L. Klein, H. Leipprand, T. Lutz, E. Krämer, Extracting the angle of attack on rotor blades from cfd simulations, Wind Energy.

[39] H. Rahimi, J. Schepers, W. Z. Shen, N. R. García, M. Schneider, D. Micallef, C. S. Ferreira, E. Jost, L. Klein, I. Herráez, Evaluation of different methods for determining the angle of attack on wind turbine blades with cfd results under axial inflow conditions, Renewable Energy 125 (2018) 866-876. 